Check for updates

Cite this: Phys. Chem. Chem. Phys., 2020, 22, 18320

Received 15th June 2020

Accepted 6th August 2020

DOI: 10.1039/d0cp03204b

rsc.li/pccp

\title{
Tube to ribbon transition in a self-assembling model peptide system
}

\author{
Axel Rüter, (D) *a Stefan Kuczera, (D) a Joakim Stenhammar, (D) a Thomas Zinn, (ID b \\ Theyencheri Narayanan (iD ${ }^{b}$ and Ulf Olsson (iD ${ }^{a}$
}

\begin{abstract}
Peptides that self-assemble into $\beta$-sheet rich aggregates are known to form a large variety of supramolecular shapes, such as ribbons, tubes or sheets. However, the underlying thermodynamic driving forces for such different structures are still not fully understood, limiting their potential applications. In the $A_{n} K$ peptide system ( $A=$ alanine, $K=$ lysine), a structural transition from tubes to ribbons has been shown to occur upon an increase of the peptide length, $n$, from 6 to 8 . In this work we analyze this transition by means of a simple thermodynamic model. We consider three energy contributions to the total free energy: an interfacial tension, a penalty for deviating from the optimal $\beta$-sheet twist angle, and a hydrogen bond deformation when the $\beta$-sheets adopt a specific self-assembled structure. Whilst the first two contributions merely provide similar constant energy offsets, the hydrogen bond deformations differ depending on the studied structure. Consequently, the tube structure is thermodynamically favored for shorter $\mathrm{A}_{n} \mathrm{~K}$ peptides, with a crossover at $n \approx 13$. This qualitative agreement of the model with the experimental observations shows, that we have achieved a good understanding of the underlying thermodynamic features within the self-assembling $A_{n} K$ system.
\end{abstract}

\section{Introduction}

The use of short peptide molecules as building blocks in materials science has become increasingly popular during the last few decades and their various applications range from cell culture scaffolds or drug delivery systems to photonic crystals and computational devices. ${ }^{1-5}$ This is, partly, due to their intrinsic biocompatibility and their ability to self-assemble into various structures such as twisted or helical ribbons, ${ }^{6,7}$ hollow nanotubes ${ }^{8,9}$ or sheets. ${ }^{10,11}$ However, to fully utilize the spontaneous self-assembly process, the ability to control the final macromolecular structure by design of the individual building blocks is crucial. ${ }^{12,13}$ Systematic studies, where small alterations to peptide model systems are made, can be used to gain fundamental thermodynamic understanding of peptide selfassembly and the underlying mechanisms. ${ }^{14}$

Peptide self-assembly is generally considered a complex process governed by a combination of non-covalent interactions, ${ }^{15,16}$ with the main driving force for aggregation being the hydrophobic interaction. ${ }^{16,17}$ Typically hydrogen bonding and electrostatic interactions between charged peptides further dictate the final internal ordering of the assemblies. ${ }^{18,19}$ The relative importance of the interactions will strongly depend on the peptide sequence

\footnotetext{
${ }^{a}$ Division of Physical Chemistry, Lund University, SE-22100 Lund, Sweden. E-mail: axel.rueter@fkem1.lu.se

${ }^{b}$ European Synchrotron Radiation Facility (ESRF), 38043 Grenoble, France
}

as well as properties of the surrounding such as temperature, $\mathrm{pH}$ and ionic strength. For example, the formation of either twisted ribbons or tubes in various self-assembling peptide systems has been shown to be dependent on the solution $\mathrm{pH}^{20-22}$ In other systems the same morphological difference was found to depend on solvent polarity ${ }^{23}$ or peptide sequence. ${ }^{24}$

The $\mathrm{A}_{n} \mathrm{~K}$ model peptide system studied in this article consists of a chain of $n$ hydrophobic alanine (A) amino acids flanked by a single lysine (K) residue as a head group. The uncapped peptide, therefore, shows a net positive charge at low $\mathrm{pH}$. Its aggregation is mainly driven by hydrophobic interaction between the alanine amino acids. ${ }^{25}$ Studies varying $n$ have revealed that shorter alanine chains $(n=6)$ assemble into hollow nanotubes, whereas $n=8,10$ peptides instead assemble into twisted ribbons. ${ }^{25-27}$ Although the macroscopic aggregate structures vary greatly, detailed analyses have shown that the arrangement of $\mathrm{A}_{n} \mathrm{~K}$ peptides within the aggregates is consistent throughout the peptide family where laminated, antiparallel $\beta$-sheets pack in a two-dimensional (2D) oblique crystal lattice regardless of the peptide length. ${ }^{28,29}$ This high internal ordering is a result of the hydrogen bonds formed between amino acids of neighboring peptides.

In this article we present high quality small- and wide-angle X-ray scattering (SAXS and WAXS, respectively) data which allow us to significantly extend the structural understanding of the two observed morphologies in the $\mathrm{A}_{n} \mathrm{~K}$ system. By identifying the different (free) energy contributions, we have developed an 
analytical model where we estimate the free energies for the two structures as a function of peptide length. The model takes into account the untwisting away from the optimal $\beta$-sheet twist, a surface tension term to account for the available hydrophobic surface area and the different hydrogen bond deformations that occur as the $\beta$-sheets adopt to the respective morphologies. These different deformations lead to different scaling behaviors of the total energy as a function of peptide length, resulting in the tube structure being energetically favorable for shorter peptides while the twisted ribbon structure is lower in free energy for longer peptides. The model shows qualitative agreement with experimental data which indicates that we likely have captured the main energy contributions within the $\mathrm{A}_{n} \mathrm{~K}$ peptide system. A fundamental thermodynamic understanding of the free energies associated with the self-assembly process can help increase the success rate in the design of novel selfassembling peptides.

\section{Results and discussion}

\subsection{Structure of peptide aggregates}

In the $\mathrm{A}_{n} \mathrm{~K}$ model peptide system the self-assembled structure is dependent on the number of alanine residues in the chain, $n$, resulting in assemblies of both tubes ${ }^{32,33}$ and twisted ribbons. $^{25,27}$ To illustrate this difference, SAXS and WAXS profiles of $\mathrm{A}_{6} \mathrm{~K}, \mathrm{~A}_{8} \mathrm{~K}$ and $\mathrm{A}_{10} \mathrm{~K}$ are presented in Fig. 1. The scattering profile of the shorter $\mathrm{A}_{6} \mathrm{~K}$ peptide shows, as expected, the characteristic features from tubular structures. ${ }^{31}$ Unlike in previous scattering experiments the scattering setup enabled very precise background measurements and, hence, the precise determination of the tube wall thickness of $3.3 \mathrm{~nm}$, corresponding roughly to the length of an extended peptide monomer, $l_{\mathrm{p}}=2.5 \mathrm{~nm}$. This verifies, that the peptides stand perpendicularly against the tube surface in a monolayer of laminated $\beta$-sheets. ${ }^{34}$ The polydispersity was determined to be $4 \%$.

The scattering data from $\mathrm{A}_{8} \mathrm{~K}$ and $\mathrm{A}_{10} \mathrm{~K}$ peptides are shown in Fig. $1 \mathrm{~B}$ and are modeled as the scattering from elliptical cylinders. ${ }^{25,27,30}$ Again, the peptide monomers are organized as a single layer of laminated $\beta$-sheets, but with a limited lamination and, thus, a limited number of $\beta$-sheets, $N \approx 15$. This monodispersity is the result of a trade-off in free energy between the hydrophobic lamination and a stretching deformation from the favorable $\beta$-sheet twist. ${ }^{27,35,36}$ Here we present data with a lower $q_{\text {min }}$ than previous reports, where $q$ is the magnitude of the scattering vector. This enabled a proper length determination of the $\mathrm{A}_{8} \mathrm{~K}$ ribbons, which were found to be roughly twice the length of the ribbons formed from $\mathrm{A}_{10} \mathrm{~K}$ peptides. Corresponding polydispersity of the minor elliptical cylinder axis is $15 \%$ for both $\mathrm{A}_{8} \mathrm{~K}$ and $\mathrm{A}_{10} \mathrm{~K}$.

Much like the critical micellar concentration in a surfactant system, the self-assembly of the $\mathrm{A}_{n} \mathrm{~K}$ peptides takes place above a certain soluble peptide volume fraction, $\phi_{\mathrm{s}}$. At a total peptide volume fraction, $\phi_{\text {tot }}>\phi_{\mathrm{s}}$, the sample contains both assembled
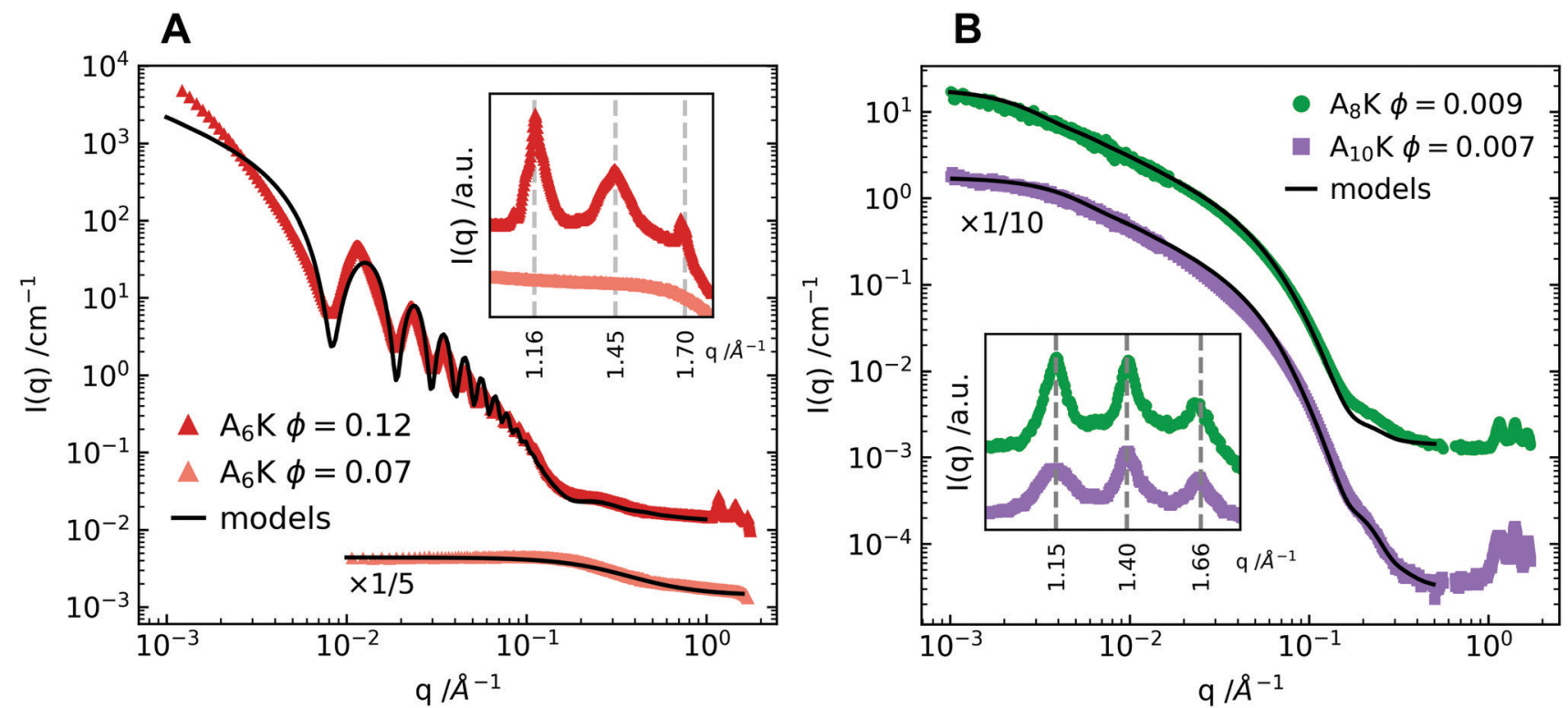

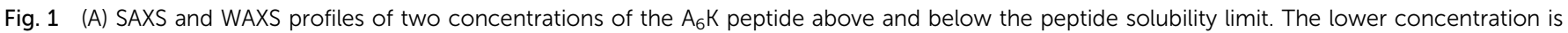

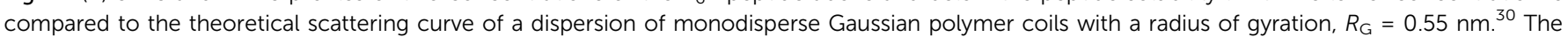

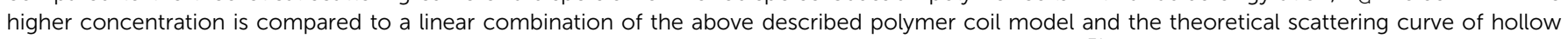

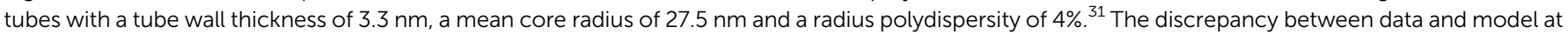

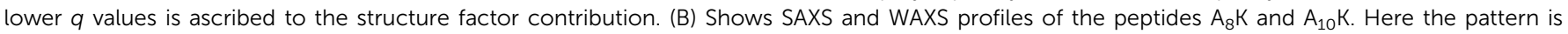

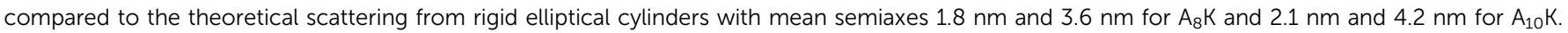

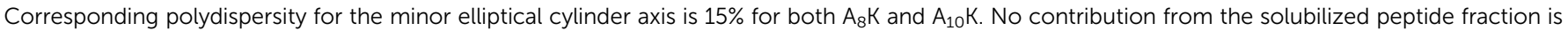

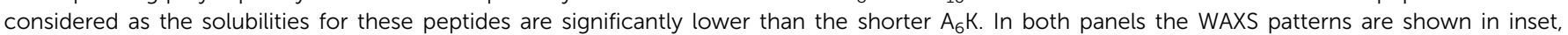
highlighting the striking similarity of the local peptide packing within the observed morphologies. 
peptides and freely dissolved peptide monomers, $\phi_{\text {tot }}=\phi_{\text {struct }}+$ $\phi_{\mathrm{s}}$. For the longer peptides $\mathrm{A}_{8} \mathrm{~K}$ and $\mathrm{A}_{10} \mathrm{~K}$, where the peptide solubility is low ${ }^{25}$ the solubilized fraction can be neglected. For $\mathrm{A}_{6} \mathrm{~K}$, on the other hand, this is not possible. In Fig. 1A, the scattering curve for $\mathrm{A}_{6} \mathrm{~K}$ at $\phi=0.07$ is shown together with the model curve for a dispersion of monodisperse Gaussian coils, ${ }^{37}$ indicating that $\phi_{\mathrm{s}} \geq 0.07$. Due to this relatively high solubilized fraction, the theoretical model of the $\mathrm{A}_{6} \mathrm{~K}$ tubes at $\phi=0.12$ includes a linear combination of the theoretical scattering from hollow tubes and a dispersion of monodisperse Gaussian polymer coils. This results in a good agreement between the scattering pattern of the tubes and the model in the intermediate to high $q$ regime.

The first maximum of the $\mathrm{A}_{6} \mathrm{~K}$ data shows a more distinguished tooth shape than $P(q)$ for the tube. This suggests a contribution from the structure factor of the tubes, which is expected, as the effective volume fraction of tubes is significantly higher than the volume fraction of peptides in the tube walls. However, there is no indication of further ordered lyotropic structures, such as hexagonal packing: this would show up as a split of the second and third order maxima as has been seen for other hollow peptide tubes. ${ }^{38}$ The position of the structure factor peak together with the upturn of intensity at low $q$ values with a power law $I(q) \sim q^{-3}$ suggests, that parallel oriented tubes are tightly packed in domains rather than homogeneously distributed due to insufficient repulsive stabilization.

Although the self-assembled structures of the $A_{n} K$ peptides differ significantly, the local peptide packing within the different aggregates is strikingly similar, as seen in the WAXS insets in Fig. 1, where the positions of the Bragg reflections are essentially the same. Moreover, both the tubes and the ribbons have previously been shown to consist of laminated $\beta$-sheets. ${ }^{25,29}$ In a previous study, the Bragg reflections in the twisted ribbons were indexed to an oblique unit cell with the lattice parameters given in Fig. 2, where the $\beta$-sheet runs parallel to $a .{ }^{28}$ A schematic of this unit cell is shown in Fig. 2. The orientation of the $\beta$-sheets within the ribbon aggregates was found to be parallel to the ribbon axis as determined by analysis of $2 \mathrm{D}$ WAXS patterns from a flow aligned sample. ${ }^{28}$

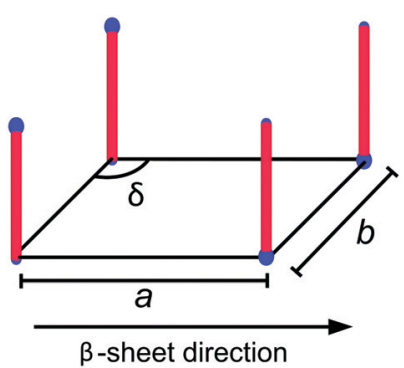

Fig. 2 A schematic of the oblique lattice with lattice parameters $a=$ $0.44 \mathrm{~nm}, b=0.54 \mathrm{~nm}$ and $\delta=100^{\circ}$ proposed as the 2D crystal unit cell of the peptides in the $\mathrm{A}_{8} \mathrm{~K}$ and $\mathrm{A}_{10} \mathrm{~K}$ twisted ribbons. ${ }^{28}$ The hydrophobic alanine residues of the peptide are here represented by a red rod, whereas the charges of the $\mathrm{N}$-terminal ammonium and the lysine side chain are represented by a smaller and a larger blue sphere, respectively.
From the similarities between the WAXS patterns the local peptide packing in the tubes and that of the ribbons are assumed to be essentially the same, i.e., in a $2 \mathrm{D}$ oblique unit cell. $^{28,29}$ 2D WAXS patterns of all three studied peptide assemblies are shown in Fig. 3. From the alignment of the $\mathrm{A}_{6} \mathrm{~K}$ tubes in the measurement cell, roughly along $q_{x}$, we conclude that the $\beta$-sheets in the tubes must run along the tube surface on a helical path at the pitch angle $\varphi=52^{\circ}$. A schematic of this is shown in Fig. 4A, where a close-up of the $\beta$-sheet shows that this helical path imposes a bending deformation to the $\beta$-sheets. We note that this value of $\varphi$ is different from what has been proposed based on solid state NMR (ssNMR) techniques. ${ }^{34}$ However, the choice of unit cell parameters in that study do not correspond to the real space distances observed in the WAXS patterns presented here (Fig. 2).

From the scattering curve of the tube system in Fig. 1A, it is evident that the tubes have strikingly uniform diameters, in accordance with previous results from cryo transmission electron microscopy. ${ }^{39}$ We note, that similar uniformity has also been reported for other systems where small self-assembled molecules form hollow nanotubes. ${ }^{38,40-42}$

In Fig. 4B we also show a schematic of the twisted ribbons formed from the $\mathrm{A}_{8} \mathrm{~K}$ and $\mathrm{A}_{10} \mathrm{~K}$ peptides, as have been characterized previously. ${ }^{25,27}$ The twisted peptide ribbons consist of $N=(2 M+1)$ laminated $\beta$-sheets, propagating along the ribbon

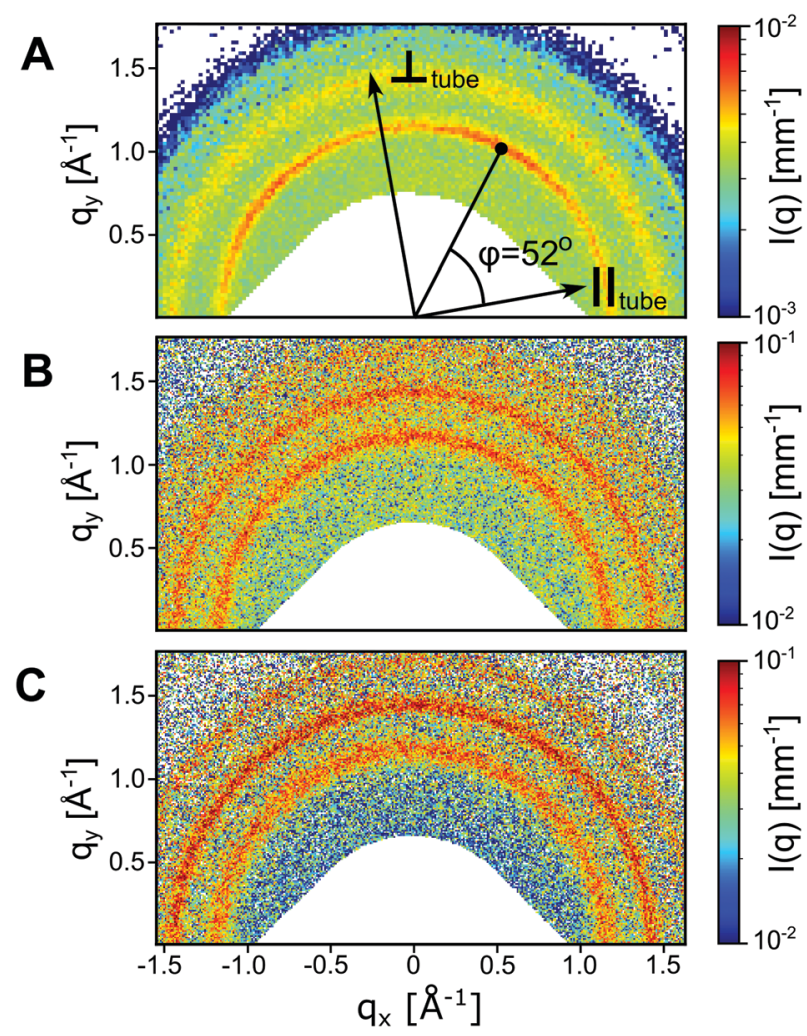

Fig. $32 \mathrm{D}$ WAXS patterns of $\mathrm{A}_{6} \mathrm{~K}$ tubes in $(\mathrm{A})$, where the parallel and perpendicular direction of the partially aligned tubes are indicated. The angle between the first reflection and the tube alignment is determined to $\varphi=52^{\circ} .2 \mathrm{D}$ WAXS patterns of $\mathrm{A}_{8} \mathrm{~K}$ and $\mathrm{A}_{10} \mathrm{~K}$ twisted ribbons are shown in (B) and (C) respectively. 

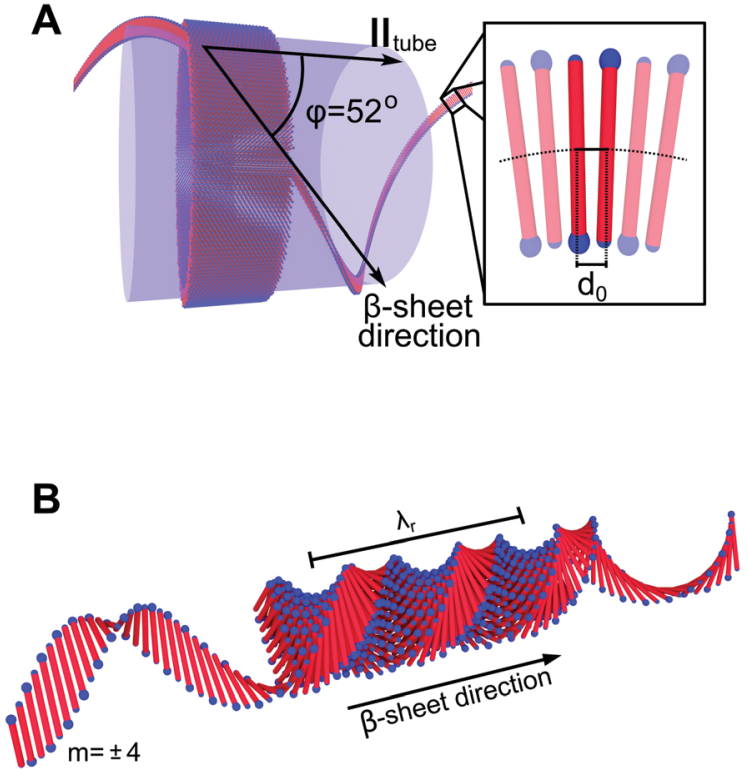

Fig. 4 (A) Schematic of the $A_{6} K$ tubes showing the helical path of the $\beta$-sheets around the tube perimeter with the helix pitch angle $\varphi=52^{\circ}$. The helical path imposes a curvature and, thus, a bending deformation of the non-twisted $\beta$-sheets. (B) A schematic image showing the twisted ribbon aggregates formed from $A_{n} \mathrm{~K}$ peptides where $n=8,10$ with a pitch $\lambda_{r}$. This specific aggregate consists of $N=9$ laminated $\beta$-sheets propagating along the ribbon length. The $\beta$-sheet $m= \pm 4$ is extended in both forward and backward direction to visualize the increased helical path of a $\beta$-sheet further out in the structure.

axis. ${ }^{25}$ Every sheet is indexed symmetrically around the central sheet $m=0$ and $M$ is the absolute value of the highest index $m$ in the structure. The ribbons were experimentally found to show a twist pitch, $\lambda_{\mathrm{r}}=16 \mathrm{~nm}$, due to the intrinsic chirality of the peptide monomers. ${ }^{27}$ Whereas the midpoints of all peptides in the central $\beta$-sheet $(m=0)$ fall on a straight line, the outer sheets $(m \neq 0)$ follow a longer helical path due to the ribbon twist. This increase in path length results in an extensional deformation of the hydrogen bonds along the helical path. In Fig. 4B the outer most $\beta$-sheet, $m= \pm 4$, has been extended in both directions to highlight the helical path.

\subsection{Peptide aggregate thermodynamic model}

As shown above, the shorter $\mathrm{A}_{n} \mathrm{~K}$ peptides prefer a hollow tube configuration while the longer instead self-assemble into twisted ribbons. Despite these macromolecular differences the local packing of peptides within the structures are strikingly similar. In the following, we will present a simple model which we use to compare the free energies of the two structures. Following the work of Nyrkova et al., ${ }^{35,36}$ we consider three contributions of the free energy: an interfacial energy, a penalty for deviating from a preferred $\beta$-sheet twist angle, and a hydrogen bond deformation when the $\beta$-sheets adopt to the self-assembled structure.

The main driving force for peptide self-assembly in water is generally considered to be the hydrophobic effect. ${ }^{16,17}$ This hydrophobic interaction drives the formation of $\beta$-sheets that aggregate further, forming laminated stacks, due to the hydrophobic interaction between the sheets. Both the $\mathrm{A}_{6} \mathrm{~K}$ nanotubes and the ribbon aggregates of $\mathrm{A}_{8} \mathrm{~K}$ and $\mathrm{A}_{10} \mathrm{~K}$ can be viewed as such laminated $\beta$-sheets, as seen in Fig. 4 , where the number of laminated $\beta$-sheets in the tubes, $N$, is practically infinite. The interfacial free energy of a $\beta$-sheet of length $L$ can be approximated as $G_{\text {int }}=\gamma 2 L l_{\mathrm{p}}$, where $\gamma$ is the water-peptide surface tension and $l_{\mathrm{p}}$ is the peptide length, assumed equal to the $\beta$-sheet width. By laminating $N$ equally long $\beta$-sheets, the number of $\beta$-sheet interfaces exposed to water is reduced by a factor $N$, which is the energetic driving force for the lamination. Using $l_{\mathrm{p}}=(n+1) d_{\mathrm{A}}$, where $d_{\mathrm{A}}=0.36 \mathrm{~nm}$ is the length of an individual amino acid, ${ }^{43}$ this interfacial energy contribution to the peptide chemical potential can be written as

$$
\mu_{\mathrm{int}}=\frac{2 \gamma(n+1) d_{\mathrm{A}} d_{0}}{N}
$$

where $d_{0}=0.44 \mathrm{~nm}^{28}$ is the $\beta$-strand separation, i.e., the peptide-peptide separation in an undeformed $\beta$-sheet. As polyalanine is strongly hydrophobic we use $\gamma=25 \mathrm{mN} \mathrm{m}^{-1}$, corresponding to $6 k_{\mathrm{B}} T \mathrm{~nm}^{-2}$. This is roughly half the value of a typical water-hydrocarbon interfacial tension. ${ }^{44}$

In the final self-assembled structure, hydrogen bonds connect the peptides to form $\beta$-sheets. ${ }^{45,46}$ Depending on the aggregate morphology these hydrogen bonds will experience a deformation from their minimum free energy. For simplicity the angular dependence on the hydrogen bond energy is neglect and each hydrogen bond of the $\beta$-sheet is assumed to have a harmonic potential:

$$
G_{\mathrm{def}}=\frac{1}{2} k_{\mathrm{def}}\left(d-d_{0}\right)^{2}
$$

where $k_{\mathrm{def}}$ is a spring constant and $G_{\mathrm{def}}$ is the potential energy of a single hydrogen bond. The peptide separation, $d$, can vary over the length of a peptide molecule and the total contribution to the chemical potential from $\beta$-sheet deformation, $\mu_{\mathrm{def}}$, is then obtained by calculating the sum of the potential energies from all $(n+1)$ hydrogen bonds along a peptide molecule.

$\beta$-Sheets are known to exhibit a left-handed twist looking along the direction of the hydrogen bonds, ${ }^{47,48}$ with an angle $\theta$ between adjacent $\beta$-strands. The twist of a long $\beta$-sheet can also be quantified by a pitch length, $\lambda_{\mathrm{r}}=2 \pi d / \theta$. Many computational studies have dealt specifically with the twist of $\beta$-sheets from polyalanine peptides, ${ }^{49-51}$ and although the driving force behind this twisting of $\beta$-sheets is not fully understood, the existence of a preferred angle, $\theta_{0}$, between adjacent $\beta$-strands is well-established. Assuming a harmonic potential, we write the contribution to the chemical potential, due to this twist deformation, as

$$
\mu_{\mathrm{twist}}=\frac{1}{2} k_{\mathrm{twist}}\left(\theta-\theta_{0}\right)^{2}
$$

where $k_{\text {twist }}$ is an elastic constant.

The total peptide chemical potential of the aggregates is the sum of the individual free energy contributions $\mu_{\text {tot }}=\mu_{\text {int }}+$ $\mu_{\mathrm{def}}+\mu_{\mathrm{twist}}$. To compare the two structures formed from the $\mathrm{A}_{n} \mathrm{~K}$ 
peptides we will now estimate the chemical potential in the two structures and how it depends on the peptide length.

Tubular structures can be considered as composed of an infinite number of laminated $\beta$-sheets, so that $\mu_{\text {int }}=0$, and $\mu_{\text {tube }}=\mu_{\text {def }}+\mu_{\text {twist }}$. As discussed above, there is a bending deformation of the $\beta$-sheets as a result of the their helical path around the tube, with an angle $\varphi$, as shown in Fig. 4A, and a corresponding pitch length, $\lambda_{\mathrm{t}}$. The $\beta$-sheets are bent with a curvature $c=R /\left(R^{2}+\lambda_{\mathrm{t}}^{2}\right)$, where $R$ is the tube radius. $R$ and $\lambda_{\mathrm{t}}$ are also related to $\varphi$ as $\tan \varphi=2 \pi R / \lambda_{\mathrm{t}}$. The amino acids are labeled with the integer number $\nu$ that runs from $-n / 2$ to $n / 2$. In the bent $\beta$-sheet $d$ varies with $\nu$ as

$$
d(\nu)=d_{0}\left(1+\nu d_{\mathrm{A}} c\right)
$$

so that the outer half of the $\beta$-sheet experiences a stretching $\left(d>d_{0}\right)$ while the inner half is compressed $\left(d<d_{0}\right)$ and where $d_{\mathrm{A}}$ is the length of an individual amino acid. A schematic visualization is shown in Fig. 5.

The twist angle, $\theta$, is essentially zero in the tube so that $\mu_{\text {twist }} \approx 0.5 k_{\text {twist }} \theta_{0}{ }^{2}$. With these considerations we arrive at

$$
\mu_{\text {tube }}=\sum_{\nu=-n / 2}^{n / 2} \frac{1}{2} k_{\text {def }}\left(d(\nu)-d_{0}\right)^{2}+\frac{1}{2} k_{\text {twist }} \theta_{0}^{2}
$$

for the peptide chemical potential in the tubes.

We have recently analyzed the twisted ribbon aggregates of $A_{8} K$ and $\mathrm{A}_{10} \mathrm{~K} .^{27}$ The ribbons were experimentally found to be rather monodisperse in width and consist of $N \approx 15$ laminated $\beta$ sheets. ${ }^{27}$ The finite value of $N$ can be understood as a compromise between the interfacial energy term, which favors infinite $N$, and the $\beta$-sheet deformation (stretching) term, due to the ribbons being twisted, that opposes lamination and instead favors $N=1 .^{27,35,36}$ The fact that the ribbons are twisted implies that $\theta_{0} \neq 0$ and that $k_{\text {twist }}$ is of significant magnitude compared to the thermal energy. The total chemical potential of the ribbons thus contains three contributions: $\mu_{\text {ribbon }}=\mu_{\text {def }}+\mu_{\text {int }}+\mu_{\text {twist }}$.

To obtain a simple expression for $\mu_{\mathrm{def}}$, the $\beta$-sheets are labeled with the integer $m$ that runs from $-(N-1) / 2$ to $(N-$ 1) $/ 2$. Furthermore, the central $\beta$-sheet, corresponding to $m=0$, is assumed to be undeformed so that $d=d_{0}$. When $m \neq 0$, on the other hand, the center of the $\beta$-sheet follows a helical path (Fig. 6), with the radius $|m| d_{\text {lam }}$ and the pitch length $\lambda_{\mathrm{r}}=2 \pi d_{0} / \theta$.

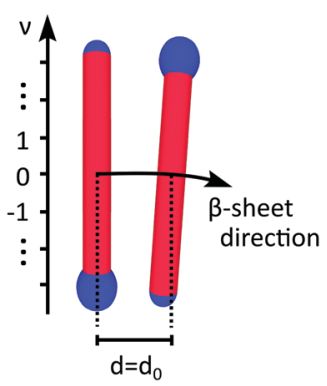

Fig. 5 Two peptides from a $\beta$-sheet within a tube, where the locations of the individual amino acids are indicated by their integer value $\nu$. The amino acid separation at $\nu=0$ for adjacent peptides is assumed $d=d_{0}$.

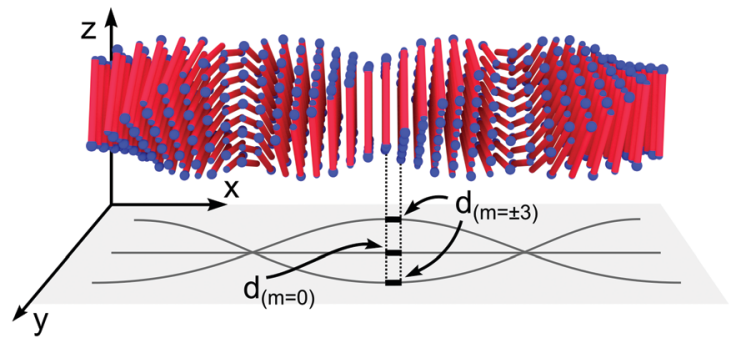

Fig. 6 A twisted ribbon with $N=9$, where the $\beta$-sheet traces of $m=-3,0$ and 3 are projected on the $x y$-plane for visualization. In a sheet where $m \neq$ 0 the amino acid separation is longer than for $m=0$.

The pitch length is independent of $m$, but as the helical radius increases with $|m|$, so does the $\beta$-sheet contour length of a pitch, $l_{\mathrm{c}}$. For this contour length we have $l_{\mathrm{c}}{ }^{2}=\left(2 \pi m d_{\mathrm{lam}}\right)^{2}+\lambda_{\mathrm{r}}{ }^{2}$. Thus, for $m \neq 0$ the $\beta$-sheets are stretched, with a $\beta$-strand separation given by

$$
d(m)=d_{0} \frac{l_{\mathrm{c}}(m)}{\lambda_{\mathrm{r}}}
$$

We then arrive at the following full expression for the peptide chemical potential in a ribbon aggregate

$$
\begin{aligned}
\mu_{\text {ribbon }}= & \frac{(n+1)}{N} \sum_{m=-\frac{(N-1)}{2}}^{\frac{(N-1)}{2}} \frac{1}{2} k_{\text {def }}\left(d(m)-d_{0}\right)^{2}+\frac{\gamma 2(n+1) d_{\mathrm{A}} d_{\mathrm{lam}}}{N} \\
& +\frac{1}{2} k_{\text {twist }}\left(\theta-\theta_{0}\right)^{2}
\end{aligned}
$$

In the first term, we multiply with $(n+1)$ because there is no bending, so that $d(m)$ is independent of the position $\nu$ along the peptide molecule. We also divide with $N$, so that $\mu_{\text {def }}$ is averaged over the different $\beta$-sheets in the ribbon.

Having now reached model expressions for $\mu_{\text {tube }}$ and $\mu_{\text {ribbon }}$ their relative stability as a function of $n$ can be compared. As can be seen from eqn (7), $\mu_{\text {ribbon }}$ increases linearly with the peptide length $(n+1)$, with an offset given by $\mu_{\text {twist }}$. In the expression for $\mu_{\text {tube }}$ (eqn (5)), on the other hand, $\mu_{\text {def }}$ has a stronger dependence to $(n+1)$. First of all $\left(d(\nu)-d_{0}\right)=\nu d_{\mathrm{A}} c$. This term is further squared and summed over the $n+1$ values of $\nu$, implying that $\mu_{\text {tube }}$ approximately scales as $(n+1)^{3}$ plus an offset given by $\mu_{\text {twist }}$. Comparing the linear scaling of $\mu_{\text {ribbon }}$ with the cubic scaling for $\mu_{\text {tube }}$ we thus expect $\mu_{\text {tube }}<\mu_{\text {ribbon }}$ for small values of $(n+1)$ and the opposite to hold for larger values of $(n+1)$.

To reach a quantitative comparison, we fix the parameters previously presented. A summary of these parameters and their values are presented in Table 1 . The values of $d_{0}, d_{\text {lam }}$ and $d_{\mathrm{A}}$ were determined experimentally, while the surface tension between peptide and water, $\gamma$, can be estimated with reasonable accuracy. However, for the parameters $k_{\text {def }}, k_{\text {twist }}$ and $\theta_{0}$, we do not have any reliable estimates. In order to check the accuracy of our model, we do therefore not perform a global analysis, but rather seek the possibility for a set of parameters than can 
Table 1 A summary of the parameters used for the calculations

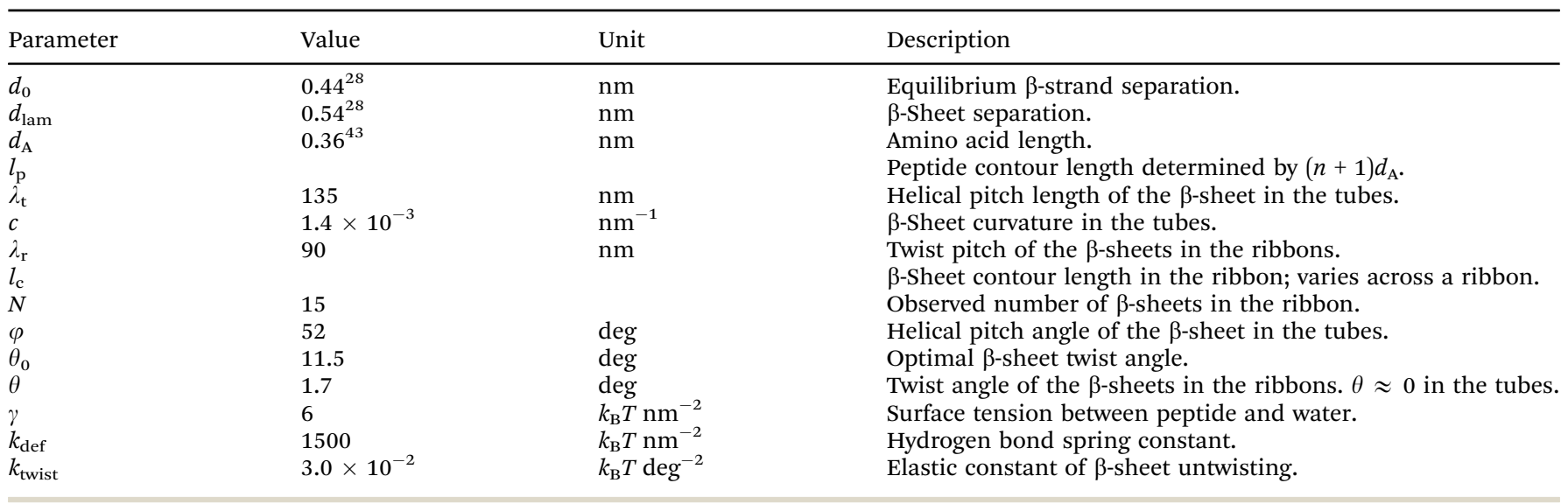

account for both the observed $N \approx 15$, and for a tube to ribbon transition reasonably close to the observed $n \approx 7$. In doing so, we have first considered the minimization of $\mu_{\text {ribbon }}$ to obtain the optimum $N$ and $\theta$ (or $\lambda_{\mathrm{r}}$ ) for given values of $\gamma, k_{\mathrm{def}}, k_{\mathrm{twist}}$ and $\theta_{0}$. In this procedure we choose $\theta_{0}=11.5^{\circ}$ and $\gamma=6 k_{\mathrm{B}} T \mathrm{~nm}^{-2}$, and then vary $k_{\mathrm{def}}$ and $k_{\mathrm{twist}}$ to find a free energy minimum for $N \approx 15$. There are of course many combinations of $k_{\text {def }}$ and $k_{\text {twist }}$ that satisfy $N \approx 15$, but one such combination of values (Table 1), results in $\theta=1.7^{\circ}$ for the ribbon, corresponding to $\lambda_{\mathrm{r}}=$ $90 \mathrm{~nm}$. This value of $\lambda_{\mathrm{r}}$ is significantly larger than the value $16 \mathrm{~nm}$ suggested from negatively stained TEM experiments. ${ }^{27}$ However, this experimental value implies an unphysical stretching compared to the ribbon width, in stark contrast to previous studies, ${ }^{52-55}$ and it is thus likely, that the value $\lambda_{\mathrm{r}}=$ $16 \mathrm{~nm}$ is underestimated due to the vacuum conditions of the TEM experiments. This is also in qualitative agreement with the computational studies, where longer $\beta$-sheets generally are associated with smaller values of $\theta_{0}{ }^{50}$

Using the presented values, we have calculated $\mu_{\text {ribbon }}$ and $\mu_{\text {tube }}$ as a function of $n$, see Fig. 7. From this analysis, we find that $\mu_{\text {tube }}<\mu_{\text {ribbon }}$ for $n<13$, i.e., a value roughly two times larger than the experimentally observed transition (between 6 and 8). While the parameters can be adjusted to yield a transition at a lower value of $n$, this semiquantitative agreement with experiments using realistic values of all parameters indicates

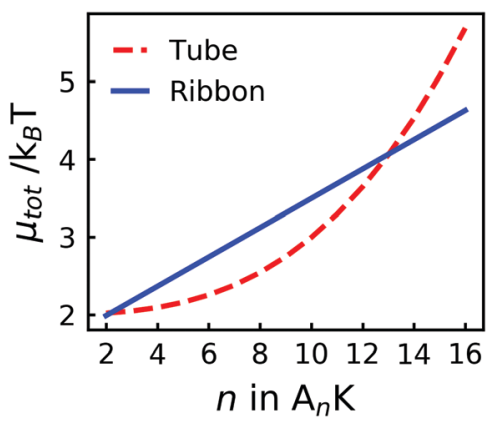

Fig. 7 The total chemical potential, $\mu_{\text {tot, }}$ for tubes and ribbons respectively showing a cross-over for $n \approx 13$. The parameters used for the calculations are presented in Table 1. that our thermodynamic model indeed captures the important ingredients behind the observed tube-to-ribbon transition. The fact that we reach this semiquantitative agreement without including a free energy contribution from electrostatic interactions, even though the peptides are known to exhibit a net positive charge, is interesting. A possible explanation for this could be a change of the protonation state of the peptide monomer upon inclusion in the aggregated structure, ${ }^{56}$ greatly reducing the charge density of the final aggregates.

We note that the tube-to-ribbon transition can be viewed as a topological transition from a helical tape to helicoids, which has been analyzed using the toolbox of differential geometry. ${ }^{57-59}$ In the tube, the $\beta$-sheet surface is bent into a cylinder with a mean curvature $H=1 /(2 R)$, but the Gaussian curvature $K=0$. The helicoid, on the other hand, is a minimal surface and characterized by $K<0$.

\section{Conclusions}

In this paper we have proposed a thermodynamic model for laminated $\beta$-sheet aggregates that predicts a transition from hollow tubes to twisted ribbons of finite width, as a function of increased peptide size. Such a transition has been observed experimentally with the alanine rich model peptides $\mathrm{A}_{n} \mathrm{~K} .{ }^{25}$ Within the model, the transition is associated with different $\beta$-sheet deformations, bending and stretching, in tubes and ribbons. The model prediction, that tube formation is favored for smaller peptides, is consistent with the general observation that peptide $\beta$-sheet nanotubes mainly form from shorter peptides, ${ }^{20,26,32,38,53,60}$ where an extreme example is the FF dipeptide. ${ }^{8}$ This indicates, that the presented thermodynamic model captures the general features of peptide self-assembly and could possibly be extended to deepen the thermodynamic insight into the self-assembled structures of molecules beyond peptide assemblies.

\section{Experimental section}

\subsection{Materials}

$\mathrm{A}_{n} \mathrm{~K}$ peptides were purchased as trifluoroacetate (tfa) stabilized salts with purities of $95 \%$ or higher from CPC Scientific Inc., and 
used without further purification. Samples were prepared by dissolution of the peptide powder in Milli-Q water. The peptide bulk densities have been previously determined, $1.45 \mathrm{~g} \mathrm{~cm}^{-3}, 1.5 \mathrm{~g} \mathrm{~cm}^{-3}$ and $1.26 \mathrm{~g} \mathrm{~cm}^{-3}$ for $\mathrm{A}_{6} \mathrm{~K}, \mathrm{~A}_{8} \mathrm{~K}$ and $\mathrm{A}_{10} \mathrm{~K}$, respectively. ${ }^{25,33}$

\subsection{Methods}

SAXS and WAXS experiments were performed on the ID02 beamline at the European Synchrotron Radiation Facility (ESRF). ${ }^{61}$ The samples were contained in a flow-through capillary cell (diameter $\sim 2 \mathrm{~mm}$ ) maintained at $25{ }^{\circ} \mathrm{C}$, which also enabled accurate background measurements from the capillary filled with water as well as flow aligning of the nanotubes. The SAXS sample-todetector distances were $8 \mathrm{~m}$ and $1.2 \mathrm{~m}$ and the WAXS detector was positioned at $0.12 \mathrm{~m}$ from the sample. The measured twodimensional scattering patterns were normalized to an absolute intensity scale after applying different detector corrections and azimuthally averaged to obtain the one-dimensional profiles. After subtraction of the corresponding normalized background, onedimensional profiles from different sample-to-detector distances were merged together to obtain the scattered intensity, $I(q)$, as a function of the magnitude of the scattering vector $q=(4 \pi / \lambda) \sin$ $\vartheta / 2$, with $\lambda$ the wavelength of the X-rays $(\simeq 1 \AA)$ and $\vartheta$ the scattering angle. The SAXS data were analyzed using the SasView software. $^{62}$

\section{Conflicts of interest}

There are no conflicts to declare.

\section{Acknowledgements}

The synchrotron experiments were performed on beamline ID02 at the European Synchrotron Radiation Facility (ESRF), Grenoble, France. This research is funded by the Knut and Alice Wallenberg Foundation, grant number KAW 2014.0052.

\section{Notes and references}

1 C. E. Semino, J. Dent. Res., 2008, 87, 606-616.

2 R. G. Ellis-Behnke, Y.-X. Liang, S.-W. You, D. K. C. Tay, S. Zhang, K.-F. So and G. E. Schneider, Proc. Natl. Acad. Sci. U. S. A., 2006, 103, 5054-5059.

3 K. Y. Lee and D. J. Mooney, Chem. Rev., 2001, 101, 1869-1880.

4 O. Berger, E. Yoskovitz, L. Adler-Abramovich and E. Gazit, Adv. Mater., 2016, 28, 2195-2200.

5 X. Zhao, F. Pan, H. Xu, M. Yaseen, H. Shan, C. A. E. Hauser, S. Zhang and J. R. Lu, Chem. Soc. Rev., 2010, 39, 3480-3498.

6 A. Aggeli, M. Bell, N. Boden, J. N. Keen, T. C. B. McLeish, I. Nyrkova, S. E. Radford and A. Semenov, J. Mater. Chem., 1997, 7, 1135-1145.

7 G. Wei, Z. Su, N. P. Reynolds, P. Arosio, I. W. Hamley, E. Gazit and R. Mezzenga, Chem. Soc. Rev., 2017, 46, 4661-4708.

8 M. Reches and E. Gazit, Science, 2003, 300, 625-627.

9 H. Xu, Y. Wang, X. Ge, S. Han, S. Wang, P. Zhou, H. Shan, X. Zhao and J. R. Lu, Chem. Mater., 2010, 22, 5165-5173.
10 H. Rapaport, G. Möller, C. M. Knobler, T. R. Jensen, K. Kjaer, L. Leiserowitz and D. A. Tirrell, J. Am. Chem. Soc., 2002, 124, 9342-9343.

11 S. Colfer, J. W. Kelly and E. T. Powers, Langmuir, 2003, 19, 1312-1318.

12 K. Rajagopal and J. P. Schneider, Curr. Opin. Struct. Biol., 2004, 14, 480-486.

13 G. M. Whitesides and B. Grzybowski, Science, 2002, 295, 2418-2421.

14 Y. Zhao, W. Yang, C. Chen, J. Wang, L. Zhang and H. Xu, Curr. Opin. Colloid Interface Sci., 2018, 35, 112-123.

15 J. Adamcik and R. Mezzenga, Angew. Chem., Int. Ed., 2018, 57, 8370-8382.

16 K. A. Dill, Biochemistry, 1990, 29, 7133-7155.

17 Y. Harano and M. Kinoshita, Chem. Phys. Lett., 2004, 399, 342-348.

18 C. N. Pace, J. M. Scholtz and G. R. Grimsley, FEBS Lett., 2014, 588, 2177-2184.

19 J. Adamcik and R. Mezzenga, Angew. Chem., Int. Ed., 2018, 57, 8370-8382.

20 K. Lu, J. Jacob, P. Thiyagarajan, V. P. Conticello and D. G. Lynn, J. Am. Chem. Soc., 2003, 125, 6391-6393.

21 A. K. Mehta, K. Lu, W. S. Childers, Y. Liang, S. N. Dublin, J. Dong, J. P. Snyder, S. V. Pingali, P. Thiyagarajan and D. G. Lynn, J. Am. Chem. Soc., 2008, 130, 9829-9835.

22 M. L. Mason, R. F. Lalisse, T. J. Finnegan, C. M. Hadad, D. A. Modarelli and J. R. Parquette, Langmuir, 2019, 35, 12460-12462.

23 Y. Zhao, L. Deng, J. Wang, H. Xu and J. R. Lu, Langmuir, 2015, 31, 12975-12983.

24 Y. Zhao, J. Wang, L. Deng, P. Zhou, S. Wang, Y. Wang, H. Xu and J. R. Lu, Langmuir, 2013, 29, 13457-13464.

25 Ç. Ç. Cenker, S. Bucak and U. Olsson, Langmuir, 2014, 30, 10072-10079.

26 I. W. Hamley, Angew. Chem., Int. Ed., 2014, 53, 6866-6881.

27 A. Rüter, S. Kuczera, D. J. Pochan and U. Olsson, Langmuir, 2019, 35, 5802-5808.

28 S. Kuczera, A. Rüter, K. Roger and U. Olsson, ChemPhysChem, 2020, 21, 1519-1523.

29 V. Castelletto, D. R. Nutt, I. W. Hamley, S. Bucak, Ç. Cenker and U. Olsson, Chem. Commun., 2010, 46, 6270-6272.

30 J. S. Pedersen, Adv. Colloid Interface Sci., 1997, 70, 171-210. 31 S. R. Kline, J. Appl. Crystallogr., 2006, 39, 895-900.

32 S. Bucak, C. Cenker, I. Nasir, U. Olsson and M. Zackrisson, Langmuir, 2009, 25, 4262-4265.

33 Ç. Ç. Cenker, S. Bucak and U. Olsson, Soft Matter, 2011, 7, 4868-4875.

34 D. A. Middleton, J. Madine, V. Castelletto and I. W. Hamley, Angew. Chem., Int. Ed., 2013, 52, 10537-10540.

35 I. A. Nyrkova, A. N. Semenov, A. Aggeli and N. Boden, Eur. Phys. J. B, 2000, 17, 481-497.

36 A. Aggeli, I. A. Nyrkova, M. Bell, R. Harding, L. Carrick, T. C. B. McLeish, A. N. Semenov and N. Boden, Proc. Natl. Acad. Sci. U. S. A., 2001, 98, 11857-11862.

37 P. Debye, J. Phys. Colloid Chem., 1947, 51, 18-32.

38 C. Valéry, M. Paternostre, B. Robert, T. Gulik-Krzywicki, T. Narayanan, J.-C. Dedieu, G. Keller, M.-L. Torres, 
R. Cherif-Cheikh, P. Calvo and F. Artzner, Proc. Natl. Acad. Sci. U. S. A., 2003, 100, 10258-10262.

39 Ç. Ç. Cenker, S. Bucak and U. Olsson, Soft Matter, 2011, 7, 4868-4875.

40 M. Gubitosi, L. Travaglini, M. C. di Gregorio, N. V. Pavel, J. Vázquez Tato, S. Sennato, U. Olsson, K. Schillén and L. Galantini, Angew. Chem., Int. Ed., 2015, 54, 7018-7021.

41 R. Mizuta, J. M. Devos, J. Webster, W. L. Ling, T. Narayanan, A. Round, D. Munnur, E. Mossou, A. A. Farahat, D. W. Boykin, W. D. Wilson, S. Neidle, R. Schweins, P. Rannou, M. Haertlein, V. T. Forsyth and E. P. Mitchell, Nanoscale, 2018, 10, 5550-5558.

42 J. Landman, S. Ouhajji, S. Prévost, T. Narayanan, J. Groenewold, A. P. Philipse, W. K. Kegel and A. V. Petukhov, Sci. Adv., 2018, 4, eaat1817.

43 G. E. Schulz and R. H. Schirmer, in Principles of Protein Structure, ed. C. R. Cantor, Springer-Verlag, 1979, ch. 5, pp. 65-107.

44 S. Zeppieri, J. Rodríguez and A. L. López de Ramos, J. Chem. Eng. Data, 2001, 46, 1086-1088.

45 T. P. Knowles, A. W. Fitzpatrick, S. Meehan, H. R. Mott, M. Vendruscolo, C. M. Dobson and M. E. Welland, Science, 2007, 318, 1900-1903.

46 G. Lamour, R. Nassar, P. H. Chan, G. Bozkurt, J. Li, J. M. Bui, C. K. Yip, T. Mayor, H. Li, H. Wu and J. A. Gsponer, Biophys. J., 2017, 112, 584-594.

47 C. Chothia, J. Mol. Biol., 1973, 75, 295-302.

48 D. W. Weatherford and F. R. Salemme, Proc. Natl. Acad. Sci. U. S. A., 1979, 76, 19-23.
49 K.-C. Chou, M. Pottle, G. Némethy, Y. Ueda and H. A. Scheraga, J. Mol. Biol., 1982, 162, 89-112.

50 A.-S. Yang and B. Honig, J. Mol. Biol., 1995, 252, 366-376.

51 L. Wang, T. O'Connell, A. Tropsha and J. Hermans, J. Mol. Biol., 1996, 262, 283-293.

52 V. Castelletto, I. Hamley, C. Cenker and U. Olsson, J. Phys. Chem. B, 2010, 114, 8002-8008.

53 V. Castelletto, I. W. Hamley, P. J. F. Harris, U. Olsson and N. Spencer, J. Phys. Chem. B, 2009, 113, 9978-9987.

54 V. Castelletto, I. W. Hamley, Ç. Cenker, U. Olsson, J. Adamcik, R. Mezzenga, J. F. Miravet, B. Escuder and F. RodriguezLlansola, J. Phys. Chem. B, 2011, 115, 2107-2116.

55 S.-T. Wang, Y. Lin, R. K. Spencer, M. R. Thomas, A. I. Nguyen, N. Amdursky, E. T. Pashuck, S. C. Skaalure, C. Y. Song, P. A. Parmar, R. M. Morgan, P. Ercius, S. Aloni, R. N. Zuckermann and M. M. Stevens, ACS Nano, 2017, 11, 8579-8589.

56 M. D. Jeppesen, P. Westh and D. E. Otzen, FEBS Lett., 2010, 584, 780-784.

57 I. A. Nyrkova and A. N. Semenov, Soft Matter, 2010, 6, 501-516. 58 R. Ghafouri and R. Bruinsma, Phys. Rev. Lett., 2005, 94, 138101. 59 J. V. Selinger, M. S. Spector and J. M. Schnur, J. Phys. Chem. $B, 2001,105,7157-7169$.

60 C. Valéry, F. Artzner and M. Paternostre, Soft Matter, 2011, 7, 9583-9594.

61 T. Narayanan, Small-Angle Scattering, in Structure from Diffraction Methods, ed. D. W. Bruce, D. O'Hare and R. I. Walton, Wiley Online Library, 2014, pp. 259-324.

62 M. Doucet, et al., SasView Version 4.1.2, Zenodo, DOI: 10.5281 /zenodo.825675. 